\title{
Analysis of complete response by MRI following neoadjuvant chemotherapy predicts pathological tumor responses differently for molecular subtypes of breast cancer
}

\author{
YUJI HAYASHI ${ }^{1,2}$, HIROYUKI TAKEI ${ }^{1}$, SATOSHI NOZU ${ }^{3}$, YOSHIHIRO TOCHIGI ${ }^{3}$, AKIHIRO ICHIKAWA ${ }^{3}$, \\ NAOKI KOBAYASHI ${ }^{3}$, MASAFUMI KUROSUMI ${ }^{4}$, KENICHI INOUE $^{5}$, TAKASHI YOSHIDA ${ }^{1}$, \\ SHIGENORI E. NAGAI ${ }^{5}$, HANAKO OBA ${ }^{4}$, TOSHIO TABEI ${ }^{5}$, JUN HORIGUCHI ${ }^{2}$ and IZUMI TAKEYOSHI ${ }^{2}$ \\ ${ }^{1}$ Division of Breast Surgery, Saitama Cancer Center, Saitama 362-0806; ${ }^{2}$ Department of Thoracic and Visceral Organ Surgery, \\ Graduate School of Medicine, Gunma University, Gunma 371-8511; Departments of ${ }^{3}$ Radiology, \\ ${ }^{4}$ Pathology and ${ }^{5}$ Breast Oncology, Saitama Cancer Center, Saitama 362-0806, Japan
}

Received August 10,2012; Accepted October 16, 2012

DOI: $10.3892 / \mathrm{ol} .2012 .1004$

\begin{abstract}
In the present study, clinical tumor response following neoadjuvant chemotherapy (NAC) was diagnosed by magnetic resonance imaging (MRI) and clinicopathological factors, including molecular subtypes at baseline, were analyzed for correlations with pathological tumor responses. In addition, clinicopathological factors were analyzed for a correlation with the MRI capacity to predict pathological complete response (pCR). Clinical tumor response evaluated by MRI following NAC was determined as a clinical CR (cCR) or a residual tumor. cCR was confirmed if no gadolinium enhancement or an enhancement equal to or less than that of glandular tissue was observed in any phase of the MRI. Pathological tumor responses following NAC were classified into grades 0 (no change) to 3 (no residual invasive cancer) according to criteria of the Japanese Breast Cancer Society. pCR was defined as grade 3 in the present study. Of 264 cases of invasive breast cancer in 260 patients (4 synchronous bilateral breast cancer cases), 59 (22\%) were diagnosed by MRI following
\end{abstract}

Correspondence to: Dr Hiroyuki Takei, Division of Breast Surgery, Saitama Cancer Center, 818 Komuro Ina, Kita-Adachi, Saitama 362-0806, Japan

E-mail: h-takei@cancer-c.pref.saitama.jp

Abbreviations: BMI, body mass index; cCR, clinical complete response; DCIS, ductal carcinoma in situ; DW, diffusion-weighted; $\mathrm{ER}$, estrogen receptor; FISH, fluorescence in situ hybridization; FN, false-negative; FP, false-positive; HER2, human epidermal growth factor receptor 2; IDC, invasive ductal carcinoma; JBCS, Japanese Breast Cancer Society; MRI, magnetic resonance imaging; NAC, neoadjuvant chemotherapy; NOS, not otherwise specified; NPV, negative predictive value; $\mathrm{pCR}$, pathological complete response; $\mathrm{PgR}$, progesterone receptor; $\mathrm{PPV}$, positive predictive value; $\mathrm{TN}$, true-negative; TP, true-positive

Key words: breast cancer, magnetic resonance imaging, clinical complete response, pathological complete response, molecular subtype
NAC as cCR and 98 (37\%) were pathologically diagnosed as pCR. In terms of predicting pCR by MRI, the sensitivity, specificity, accuracy, positive predictive value (PPV) and negative predictive value (NPV) were 44, 90, 73, 73 and $73 \%$, respectively. Tumor size, hormone receptor status, human epidermal growth factor receptor 2 (HER2) status, molecular subtype and histological type were significantly correlated with pathological tumor responses. pCR rates increased in the following order: luminal/HER2-negative (14\%), luminal/HER2-positive (32\%), triple-negative (46\%) and non-luminal/HER2-positive $(73 \%)$ tumors. Sensitivity and specificity were the highest (60 and $100 \%$, respectively) in triple-negative tumors. PPV decreased in the following order: triple-negative (100\%), non-luminal/HER2-positive $(92 \%)$, luminal/HER2-positive (46\%) and luminal/HER2-negative (33\%) tumors. In conclusion, MRI evaluation is useful for predicting pCR following NAC, particularly for triple-negative tumors.

\section{Introduction}

Neoadjuvant chemotherapy (NAC) is a standard treatment for locally advanced breast cancer. Previous randomized controlled trials revealed that NAC increased the rate of breast-conserving surgery (BCS) and provided the same survival benefits as postoperative chemotherapy $(1,2)$. To this end, NAC has become a standard treatment for females with operable breast cancer who request BCS, even when a mastectomy was suitable at presentation.

In breast cancer patients treated with NAC, survival rates are significantly improved in patients with a pathological complete response (pCR) than in patients with a residual tumor $(2,3)$. Furthermore, pCR rates have recently increased owing to development of novel chemotherapy regimens $(2,4,5)$.

Theoretically, surgery is not required for patients whose tumors have been completely eradicated by NAC. However, eradication of the tumor is only determined by pathological analysis of the surgically-removed breast tissue that may have contained the tumor. The development of a diagnostic 
tool capable of precisely predicting $\mathrm{pCR}$ following NAC, may enable surgery to be omitted or minimized.

Core needle biopsy is an example of such a tool. Additional options are non-invasive imaging methods, including mammography, ultrasound, magnetic resonance imaging (MRI) and computed tomography (CT). Previously, MRI was analyzed for assessment of tumor responses following NAC and was demonstrated to be more reliable than mammography or ultrasound $(6,7)$.

In the most recent study, pCR was differently observed between various molecular subtypes of breast cancer classifications based on estrogen receptor (ER), progesterone receptor $(\mathrm{PgR})$ and human epidermal growth factor receptor 2 (HER2) (8). To predict pCR following NAC, evaluation of clinical tumor response by MRI, as well as the molecular subtype of the tumor, is important. In the present study, clinical tumor response diagnosed by MRI and clinicopathological factors at baseline were analyzed for correlations with pathological tumor responses following NAC. In addition, clinicopathological factors, including molecular subtypes, were analyzed for a correlation with the MRI capacity to predict pCR.

\section{Materials and methods}

Patients. Female patients diagnosed with invasive breast cancer by core needle biopsy were treated with NAC. Following NAC, patients were examined using MRI at the Saitama Cancer Center (Saitama, Japan). Patients underwent surgery at the same institute between February 2003 and June 2008.

Of the 2427 breast cancer cases treated with surgery between February 2003 and June 2008 at the center, 384 (15.8\%) were treated with NAC. Of these 384 cases, 120 that did not undergo MRI following NAC were excluded, leaving 264 breast cancer cases of 260 patients (4 synchronous bilateral) eligible for inclusion in the present study. The study was approved by the ethics committee of Saitama Cancer Center, Saitama, Japan. Written informed patient consent was obtained from the patient.

MRI technique. Details of the MRI technique are presented in Table I. Briefly, the device used was an Intera Achieva Nova Dual 1.5 T instrument (Philips, Aachen, Germany) with a SENSE body coil. Transverse images were obtained by diffusion-weighted (DW) imaging. Coronal images were obtained by contrast-enhanced dynamic imaging. Sagittal images were obtained by contrast-enhanced late-phase imaging. Additionally, depending on the case, sagittal images were obtained by $\mathrm{T} 2$-weighted fat-suppressed imaging prior to infusion of contrast material.

Clinical tumor response evaluated by MRI. Physicians evaluated clinical tumor responses prior to surgery by physical examination, mammography and ultrasound. Radiologists evaluated clinical tumor response following NAC by MRI. MRI diagnoses were performed independently of physical examination, mammography or ultrasound observations. In the present study, clinical tumor response diagnosed by MRI following NAC was defined as a clinical complete response (cCR) or as a residual tumor. cCR was diagnosed if no gadolinium enhancement or an enhancement equal to or less than that of glandular tissue was observed in any phase of the MRI.

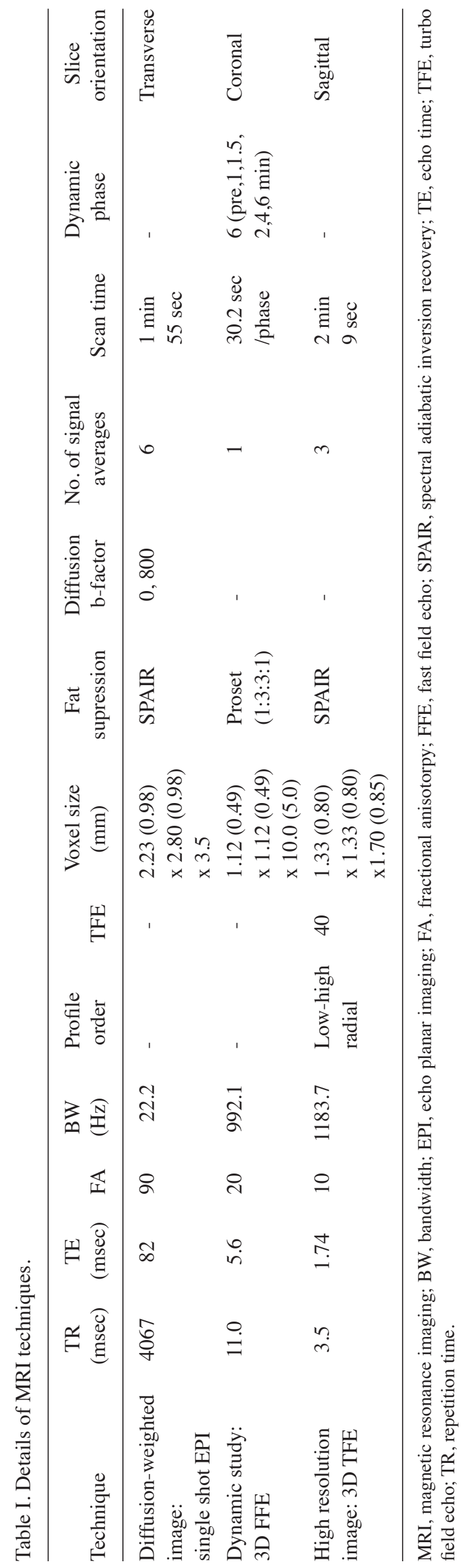




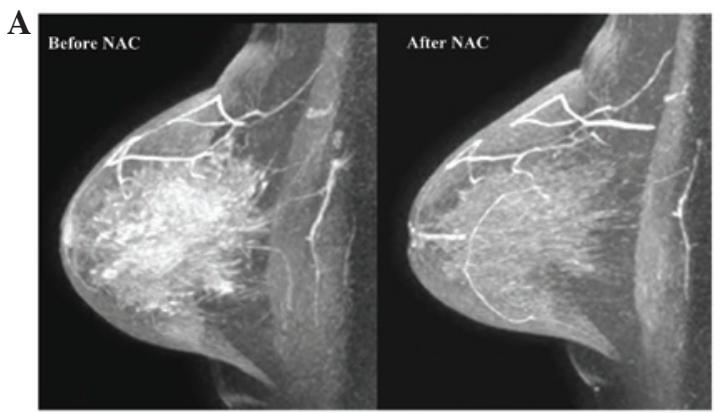

B

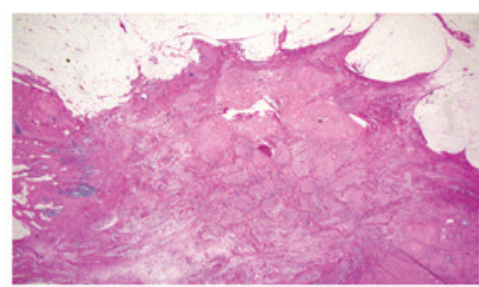

C

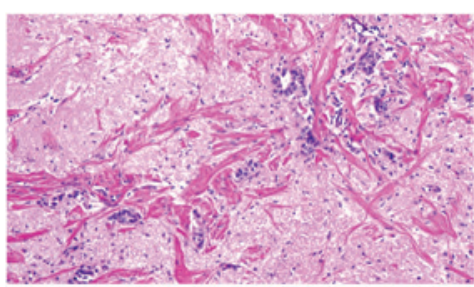

Figure 1. A false-positive case of magnetic resonance imaging (MRI) predicting a pathological complete response. (A) No gadolinium enhancement was detected by MRI. (B and C) Histological analysis indicates scattered distribution of invasive breast cancer cells was identified in a scarred area (magnification, $\mathrm{x} 2$ and $\mathrm{x} 20$, respectively). In this case, pathological tumor response was determined as grade 2 according to the Japanese Breast Cancer Society criteria.

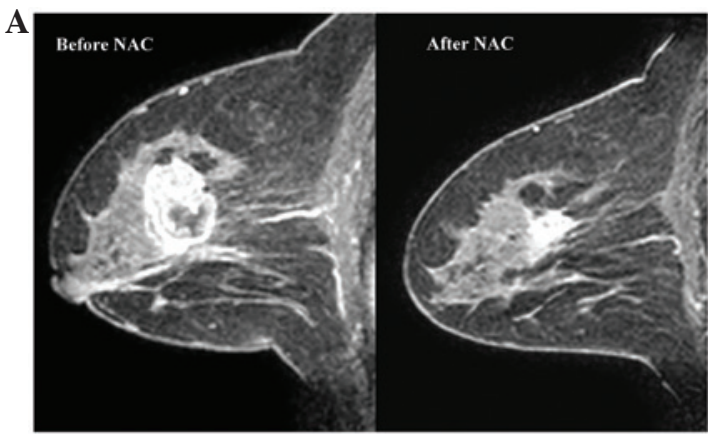

B

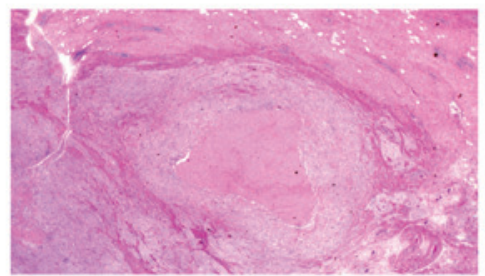

C

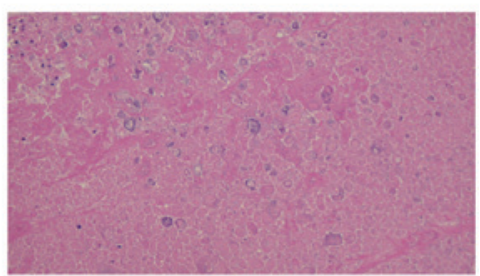

Figure 2. A false-negative case of magnetic resonance imaging (MRI) predicting a pathological complete response. (A) Gadolinium enhancement was detected by MRI. (B and C) No cancer cells were identified in the scarred area (magnification, $\mathrm{x} 2$ and $\mathrm{x} 20$, respectively).

Table II. Pathological tumor response and MRI diagnosis.

\begin{tabular}{lccccc}
\hline & \multicolumn{4}{l}{ Pathological tumor response, $\mathrm{n}(\%)$} & \\
\cline { 2 - 5 } Response & 0 & 1 & 2 & 3 & Total $(\%)$ \\
\hline cCR & $0(0.0)$ & $1(1.7)$ & $15(25.4)$ & $43(72.9)$ & $59(22.3)$ \\
$\begin{array}{l}\text { Residual } \\
\text { tumor }\end{array}$ & $4(2.0)$ & $80(39.0)$ & $66(32.2)$ & $55(26.8)$ & $205(77.7)$ \\
Total & $4(1.5)$ & $81(30.7)$ & $81(30.7)$ & $98(37.1)$ & $264(100.0)$ \\
\hline
\end{tabular}

$\mathrm{P}<0.0001$. cCR, clinical complete response; MRI, magnetic resonance imaging.

Presence of a residual tumor was diagnosed if an enhancement more than that of glandular tissue was identified in any phase of the MRI. A case of cCR and residual tumour are presented in Fig. 1A and 2A, respectively.

Pathological tumor responses. Pathological tumor response following NAC was evaluated by pathologists according to the criteria of the Japanese Breast Cancer Society (JBCS) (9) and were classified as grade 0 (no change), 1 (moderate change), 2 (marked change) and 3 (pCR). In the present study, a pCR was determined to be grade 3 according to the JBCS criteria, defined as the absence of residual invasive cancer irrespective of the presence or absence of residual ductal carcinoma in situ (DCIS).
Correlation between tumor response evaluated by MRI and pathology. A case of accurate prediction of $\mathrm{pCR}$ by MRI was defined as a cCR diagnosed by MRI and a pCR by pathology (true-positive, TP). A case of accurate prediction of a residual tumor by MRI was defined as a residual tumor diagnosed by MRI and pathology (true-negative, TN). A case of inaccurate prediction of $\mathrm{pCR}$ by MRI was defined as a cCR diagnosed by MRI but a residual tumor diagnosed by pathology (false-positive, FP). A case of inaccurate prediction of a residual tumor by MRI was defined as a residual tumor diagnosed by MRI but a pCR diagnosed by pathology (false-negative, FN). Based on these parameters, sensitivity, specificity, accuracy, positive predictive value (PPV) and negative predictive value (NPV) were calculated.

To analyze how accurately MRI predicts the absence or presence of residual DCIS without invasive cancer following NAC, MRI diagnoses were analyzed for a correlation with the presence or absence of residual DCIS in tumors for which the pCR was diagnosed.

Clinicopathological factors. Patient age and body mass index (BMI) at MRI evaluation; clinical tumor size and clinical nodal status at baseline; histological types; ER, PgR and HER 2 expression at baseline; and molecular subtypes at baseline were analyzed for correlations with pathological tumor response and the TP, TN, FP and FN rates for predicting pCR by MRI. The clinical tumor size and nodal status at baseline were classified according to the criteria of the American Joint Committee on Cancer (10). 
Table III. Pathological tumor response and clinicopathological factors.

\begin{tabular}{|c|c|c|c|c|c|c|}
\hline \multirow[b]{2}{*}{ Clinicopathological factors } & \multicolumn{4}{|c|}{ Pathological tumor response (\%) } & \multirow[b]{2}{*}{ Total } & \multirow[b]{2}{*}{ P-value } \\
\hline & 0 & 1 & 2 & 3 & & \\
\hline \multicolumn{7}{|l|}{ Age (years) } \\
\hline$\leq 50$ & $3(2.2)$ & $43(32.1)$ & $44(32.8)$ & $44(32.8)$ & 134 & \multirow[t]{2}{*}{0.41} \\
\hline$\geq 51$ & $1(0.8)$ & $38(29.2)$ & $37(28.5)$ & $54(41.5)$ & 130 & \\
\hline \multicolumn{7}{|l|}{ Body mass index } \\
\hline$\leq 23$ & $1(0.7)$ & $43(30.7)$ & $39(27.9)$ & $57(40.7)$ & 140 & \multirow[t]{2}{*}{0.37} \\
\hline$>23$ & $3(2.5)$ & $36(29.5)$ & $42(34.4)$ & $41(33.6)$ & 122 & \\
\hline \multicolumn{7}{|l|}{ Clinical tumor size } \\
\hline $\mathrm{T} 1$ & $1(8.3)$ & $2(16.7)$ & $0(0.0)$ & $1(8.3)$ & 12 & \multirow[t]{4}{*}{0.046} \\
\hline $\mathrm{T} 2$ & $2(1.3)$ & $39(25.5)$ & $44(28.8)$ & $68(44.4)$ & 153 & \\
\hline $\mathrm{T} 3$ & $0(0.0)$ & $27(42.9)$ & $20(31.7)$ & $16(25.4)$ & 63 & \\
\hline $\mathrm{T} 4$ & $1(2.8)$ & $13(36.1)$ & $13(36.1)$ & $9(25.0)$ & 36 & \\
\hline \multicolumn{7}{|l|}{ Clinical nodal status } \\
\hline No & $3(4.8)$ & $16(25.4)$ & $15(23.8)$ & $29(46.0)$ & 63 & \multirow[t]{4}{*}{0.11} \\
\hline N1 & $1(0.7)$ & $46(32.2)$ & $44(30.8)$ & $52(36.4)$ & 143 & \\
\hline N2 & $0(0.0)$ & $10(32.3)$ & $9(29.0)$ & $12(38.7)$ & 31 & \\
\hline $\mathrm{N} 3$ & $0(0.0)$ & $9(33.3)$ & $13(48.1)$ & $5(18.5)$ & 27 & \\
\hline \multicolumn{7}{|l|}{ Estrogen receptor } \\
\hline Negative & $2(1.7)$ & $26(22.6)$ & $18(15.7)$ & $69(60.0)$ & 115 & \multirow[t]{2}{*}{$<0.0001$} \\
\hline Positive & $2(1.3)$ & $55(36.9)$ & $63(42.3)$ & $29(19.5)$ & 149 & \\
\hline \multicolumn{7}{|l|}{ Progesterone receptor } \\
\hline Negative & $3(2.1)$ & $35(24.6)$ & $26(18.3)$ & $78(54.9)$ & 142 & \multirow[t]{2}{*}{$<0.0001$} \\
\hline Positive & $1(0.8)$ & $46(37.8)$ & $55(45.1)$ & $20(16.4)$ & 122 & \\
\hline \multicolumn{7}{|l|}{ HER 2} \\
\hline 0 & $2(5.3)$ & $15(39.5)$ & $16(42.1)$ & $5(13.2)$ & 38 & \multirow[t]{4}{*}{$<0.0001$} \\
\hline $1+$ & $2(2.6)$ & $29(38.2)$ & $24(31.6)$ & $21(27.6)$ & 76 & \\
\hline $2+$ & $0(0.0)$ & $25(39.7)$ & $17(27.0)$ & $21(33.3)$ & 63 & \\
\hline $3+$ & $0(0.0)$ & $12(13.8)$ & $24(27.6)$ & $51(58.6)$ & 87 & \\
\hline \multicolumn{7}{|l|}{ Molecular subtype } \\
\hline Luminal/HER2-negative & $2(2.2)$ & $37(39.8)$ & $41(44.1)$ & $13(14.0)$ & 93 & \multirow[t]{4}{*}{$<0.0001$} \\
\hline Luminal/HER2-positive & $0(0.0)$ & $15(27.8)$ & $22(40.7)$ & $17(31.5)$ & 54 & \\
\hline Non-luminal/ HER2-positive & $0(0.0)$ & $7(10.6)$ & $11(16.7)$ & $48(72.7)$ & 66 & \\
\hline Triple-negative & $2(4.5)$ & $17(38.6)$ & $5(11.4)$ & $20(45.5)$ & 44 & \\
\hline \multicolumn{7}{|l|}{ Histological type } \\
\hline IDC NOS & $3(1.2)$ & $73(29.6)$ & $74(30.0)$ & $97(39.3)$ & 247 & \multirow[t]{3}{*}{0.026} \\
\hline Others & $1(5.9)$ & $8(82.4)$ & $7(47.1)$ & $1(5.9)$ & 17 & \\
\hline Total & $4(1.5)$ & $81(30.7)$ & $81(30.7)$ & $98(37.1)$ & 264 & \\
\hline
\end{tabular}

HER2, human epidermal growth factor receptor 2; IDC, invasive ductal carcinoma; NOS, not otherwise specified. A total of two and seven cases were missing data on body mass index and molecular subtype, respectively.

Histological types were divided into two categories: invasive ductal carcinoma (IDC) not otherwise specified and other types of IDC. The expression of ER and PgR at baseline was measured by immunohistochemistry or an enzyme immunoassay. Cut-off values for ER and PgR were $\geq 10 \%$ positive cells by immunohistochemistry or $\geq 10 \mathrm{fmol} / \mathrm{mg}$ protein by the enzyme immunoassay. HER2 expression at baseline was measured by immunohistochemistry using Herceptest ${ }^{\mathrm{TM}}$ (Dako, Glostrup, Denmark) and was classified as $0,1+, 2+$ or 3+. HER2 2+ was further divided into positive and negative for HER2 by fluorescence in situ hybridization (FISH) using PathVysion ${ }^{\circledR}$ HER-2 DNA probe kit (Abbott Molecular, Wiesbaden, Germany). The HER2-positive status was defined as HER $23+$ by immunohistochemistry or HER 2 gene amplification by FISH.

Molecular subtypes at baseline were classified into four categories based on the status of ER, PgR and HER2. Luminal/HER2-negative tumors were positive for the ER 
Table IV. Number and ratio of TP, TN, FP and FN cases and clinicopathological factors.

\begin{tabular}{|c|c|c|c|c|c|c|}
\hline Clinicopathological factors & $\mathrm{TP}(\%)$ & $\mathrm{TN}(\%)$ & $\mathrm{FP}(\%)$ & $\mathrm{FN}(\%)$ & Total & P-value \\
\hline \multicolumn{7}{|l|}{ Age (years) } \\
\hline$\leq 50$ & $20(14.9)$ & $78(58.2)$ & $12(9.0)$ & $24(17.9)$ & 134 & \\
\hline$\geq 51$ & $23(17.7)$ & $72(55.4)$ & $4(3.1)$ & $31(23.8)$ & 130 & 0.15 \\
\hline \multicolumn{7}{|l|}{ Body mass index } \\
\hline$\leq 23$ & $24(17.1)$ & $76(54.3)$ & $7(5.0)$ & $33(23.6)$ & 140 & \\
\hline$>23$ & $19(15.6)$ & $72(59.0)$ & $9(7.4)$ & $22(18.0)$ & 122 & 0.59 \\
\hline \multicolumn{7}{|l|}{ Clinical tumor size } \\
\hline $\mathrm{T} 1$ & $4(33.3)$ & $7(58.3)$ & $0(0.0)$ & $1(8.3)$ & 12 & \\
\hline $\mathrm{T} 2$ & $33(21.6)$ & $76(49.7)$ & $9(5.9)$ & $35(22.9)$ & 153 & \\
\hline $\mathrm{T} 3$ & $4(6.3)$ & $42(66.7)$ & $5(7.9)$ & $12(19.0)$ & 63 & \\
\hline $\mathrm{T} 4$ & $2(5.6)$ & $25(69.4)$ & $2(5.6)$ & $7(19.4)$ & 36 & 0.048 \\
\hline \multicolumn{7}{|l|}{ Clinical nodal status } \\
\hline NO & $16(25.4)$ & $30(47.6)$ & $4(6.3)$ & $13(20.6)$ & 63 & \\
\hline $\mathrm{N} 1$ & $23(16.1)$ & $83(58.0)$ & $8(5.6)$ & $29(20.3)$ & 143 & \\
\hline $\mathrm{N} 2$ & $2(6.5)$ & $18(58.1)$ & $1(3.2)$ & $10(32.3)$ & 31 & \\
\hline $\mathrm{N} 3$ & $2(7.4)$ & $19(70.4)$ & $3(11.1)$ & $3(11.1)$ & 27 & 0.16 \\
\hline \multicolumn{7}{|l|}{ Estrogen receptor } \\
\hline Negative & $34(29.6)$ & $44(38.3)$ & $2(1.7)$ & $35(30.4)$ & 115 & \\
\hline Positive & $9(6.0)$ & $106(71.1)$ & $14(9.4)$ & $20(13.4)$ & 149 & $<0.0001$ \\
\hline \multicolumn{7}{|l|}{ Progesterone receptor } \\
\hline Negative & $39(27.5)$ & $60(42.3)$ & $4(2.8)$ & $39(27.5)$ & 142 & \\
\hline Positive & $4(3.3)$ & $90(73.8)$ & $12(9.8)$ & $16(13.1)$ & 122 & $<0.0001$ \\
\hline \multicolumn{7}{|l|}{ HER 2} \\
\hline 0 & $3(7.9)$ & $33(86.8)$ & $0(0.0)$ & $2(5.3)$ & 38 & \\
\hline $1+$ & $8(10.5)$ & $50(65.8)$ & $5(6.6)$ & $13(17.1)$ & 76 & \\
\hline $2+$ & $9(14.3)$ & $36(57.1)$ & $6(9.5)$ & $12(19.0)$ & 63 & \\
\hline $3+$ & $23(26.4)$ & $31(35.6)$ & $5(5.7)$ & $28(32.2)$ & 87 & $<0.0001$ \\
\hline \multicolumn{7}{|l|}{ Molecular subtype } \\
\hline Luminal/HER2-negative & $4(4.3)$ & $72(77.4)$ & $8(8.6)$ & $9(9.7)$ & 93 & \\
\hline Luminal/HER2-positive & $5(9.3)$ & $31(57.4)$ & $6(11.1)$ & $12(22.2)$ & 54 & \\
\hline Non-luminal/ HER2-positive & $22(33.3)$ & $16(24.2)$ & $2(3.0)$ & $26(39.4)$ & 66 & \\
\hline Triple-negative & $12(27.3)$ & $24(54.5)$ & $0(0.0)$ & $8(18.2)$ & 44 & $<0.0001$ \\
\hline \multicolumn{7}{|l|}{ Histological type } \\
\hline IDC NOS & $43(17.4)$ & $136(55.1)$ & $14(5.7)$ & $54(21.9)$ & 247 & \\
\hline Others & $0(0.0)$ & $14(82.4)$ & $2(11.8)$ & $1(5.9)$ & 17 & 0.047 \\
\hline Total & $43(16.3)$ & $150(56.8)$ & $16(6.1)$ & $55(20.8)$ & 264 & \\
\hline
\end{tabular}

HER2, human epidermal growth factor receptor 2; IDC, invasive ductal carcinoma; NOS, not otherwise specified. TP, true-positive; TN, true-negative; FP, false-positive; FN, false-negative. A total of two and seven cases were missing data on body mass index and molecular subtype, respectively.

and/or PgR and negative for HER2. Luminal/HER2-positive tumors were positive for the ER and/or PgR and HER2. Non-luminal/HER2-positive tumors were negative for the ER and PgR and positive for HER2. Triple-negative tumors were defined as negative for the ER, PgR and HER2.

Statistical analysis. Statistical analysis was performed using Fisher's exact test. $\mathrm{P}<0.05$ were considered to indicate a statistically significant difference. Analysis was performed using Statview-J 4.5® (Abacus Concepts, Piscataway, NJ, USA).

\section{Results}

Patients age ranged between 23 and 71 years (mean, 51 years). Of 264 breast cancer cases, 59 (22\%) and 205 (78\%) were diagnosed by MRI following NAC as a cCR or residual tumor, respectively (Table II). Four (2\%), 81 (31\%), 81 (31\%) and 98 (37\%) were pathologically diagnosed as grade $0,1,2$ and 3 (pCR), respectively (Table II).

Of the clinicopathological factors, clinical T category, ER, PgR, HER2, molecular subtype and histological type at base- 
Table V. pCR rate, sensitivity, specificity, accuracy, PPV and NPV for predicting pCR by MRI and molecular subtypes.

\begin{tabular}{lccccrr}
\hline Molecular subtype & pCR rate & Sensitivity & Specificity & Accuracy & PPV & NPV \\
\hline Luminal/HER2-negative & 14.0 & 30.8 & 90.0 & 81.7 & 33.3 & 88.9 \\
Luminal/HER2-positive & 31.5 & 29.4 & 83.8 & 66.7 & 45.5 & 72.1 \\
Non-luminal/ HER2-positive & 72.7 & 45.8 & 88.9 & 57.6 & 91.7 & 38.1 \\
Triple-negative & 45.5 & 60.0 & 100.0 & 81.8 & 100.0 & 75.0 \\
Total & 37.1 & 43.9 & 90.4 & 73.1 & 72.9 & 73.2 \\
\hline
\end{tabular}

All values are presented as percentages. HER2, human epidermal growth factor receptor 2; NPV, negative predictive value; pCR, pathological complete response; PPV, positive predictive value; MRI, molecular resonance imaging.

Table VI. Presence or absence of residual DCIS and MRI diagnoses.

\begin{tabular}{lrrr}
\hline & \multicolumn{2}{c}{ Residual DCIS (\%) } & \\
\cline { 2 - 3 } Response & Absence & Presence & Total (\%) \\
\hline cCR & $36(81.8)$ & $8(18.2)$ & $44(44.9)$ \\
Residual tumor & $30(55.6)$ & $24(44.4)$ & $54(55.1)$ \\
Total & $66(67.3)$ & $32(32.7)$ & $98(100.0)$ \\
\hline
\end{tabular}

$\mathrm{P}=0.0058$. cCR, clinical complete response; DCIS, ductal carcinoma in situ; MRI, magnetic resonance imaging.

line were identified to significantly correlate with pathological tumor response (Table III). However, clinical N category at baseline and age and BMI at MRI evaluation did not correlate with these responses (Table III).

In terms of predicting pCR by MRI, 43 (16\%), 150 (57\%), $16(6 \%)$ and $55(21 \%)$ were TP, TN, FP and FN, respectively (Table IV). Clinical T category, ER, PgR, HER2, molecular subtype and histological type at baseline were identified to significantly correlate with ratio of TP, TN, FP and FN cases (Table IV). However, clinical N category at baseline and age and BMI at MRI evaluation did not correlate with this ratio (Table IV).

Based on the number of TP, TN, FP and FN cases, the sensitivity, specificity, accuracy, PPV and NPV were 44, 90, 73,73 and $73 \%$, respectively, in all cases (Table V). MRI FP and FN cases are presented in Figs. 1 and 2, respectively.

The pCR rates differed between the 4 molecular subtypes (Tables III and V). pCR rates increased from $14 \%$ to $73 \%$ in the order, luminal/HER2-negative, luminal/HER2-positive, triple-negative and non-luminal/HER2-positive tumors. The sensitivity, specificity, accuracy, PPV and NPV for predicting $\mathrm{pCR}$ differed between these subtypes (Table V). Sensitivity and specificity were the highest (60 and 100\%, respectively) in triple-negative tumors. The accuracy was the highest (82\%) in luminal/HER2 negative and triple-negative tumors. PPV decreased in order from triple-negative (100\%), non-luminal/HER2-positive (92\%), luminal/HER2-positive $(46 \%)$ and luminal/HER2-negative (33\%) tumors. NPV was the highest (89\%) in luminal/HER2-negative tumors and the lowest (38\%) in non-luminal/HER2-positive tumors.
In 98 pCR cases, 66 (67\%) had no residual DCIS and 32 (33\%) had residual DCIS (Table VI). In terms of predicting a lack of residual DCIS by MRI, 36 (37\%), 24 (25\%), 8 (8\%) and $30(31 \%)$ cases were diagnosed as TP, TN, FP and FN, respectively. The sensitivity, specificity, accuracy, PPV and NPV were $55,75,61,82$ and $44 \%$, respectively.

\section{Discussion}

In the present study, a cCR diagnosed by MRI was identified in 59 (22\%) of 264 cases of breast cancer treated with NAC. Of the $59 \mathrm{cCR}$ cases, 43 were TP and 16 were FP. The number of MRI FP cases was low (6\%) in all cases. Of the 16 MRI FP cases, 15 were pathologically diagnosed as a grade 2 response and one was diagnosed as a grade 1 response. According to the JBCS criteria, a grade 2 response is indicative of marked changes in two-thirds or more of the whole tumor area (grade 2A) or only a few cancer cells remain (grade 2B) (9). Therefore, in this study, the majority of cases diagnosed as a cCR by MRI were considered to have responded well to NAC.

The FP MRI may be explained by the hypothesis that residual invasive cancer does not require vasculature due to their small tumor volumes or a low viability of cancer cells. In the present study, MRI FP rates were higher in ER- or PgR-positive tumors compared with ER- or PgR-negative tumors, indicating that hormone receptor-positive tumors survive with a lower vasculature following NAC than hormone receptor negative-tumors.

Chen et al (11) reported a PPV of $74 \%$ using the same criteria for cCR and pCR as the present study. This result is almost identical to the result obtained in our study (73\%). In addition, the authors reported that the PPV increased in HER2-positive (95\%), compared with HER2-negative (50\%) tumors. These results are also consistent with current results which indicate that the PPV was $33 \%$ in luminal/HER2-negative tumors and 92\% in non-luminal/HER2-positive tumors. In an additional study by the same group (12) mean MRI-pathological size discrepancy was identified to be smaller in HER2-positive and hormone receptor-negative tumors or tumors with high Ki67 scores compared with their counterparts. In addition, among HER2-negative tumors, the accuracy of MRI for predicting pCR was increased in hormone receptor-negative cancers compared with hormone receptor positive-cancers and was also improved in high-proliferation tumors compared with low-proliferation tumors (13). In another study, MRI more accurately predicted tumor sizes following NAC in HER2-positive and triple-nega- 
tive tumors compared with luminal tumors (14). By contrast, De Los Santos et al (15) reported that the molecular subtype was not identified to significantly affect the sensitivity, specificity, PPV or NPV of MRI for the prediction of pathological responses. However, the number of patients analyzed in the study was smaller than that of the present study. Current results indicate that MRI is more useful for predicting pCR following NAC in patients with non-luminal/HER2-positive tumors or in those with triple-negative tumors compared with patients with luminal/HER2-negative tumors.

In the current study, the number of MRI FN cases was $\sim 1 / 5(21 \%)$ of all cases and the ratio increased with increased HER2 scores and it was higher in ER- or PgR-negative tumors compared to ER- or PgR-positive tumors. In 4 molecular subtypes, the number of MRI FN cases was lowest (10\%) in luminal/HER2-negative tumors and highest (39\%) in non-luminal/HER2-positive tumors. These results indicate that HER2 overexpression or hormone receptor negativity appears to demonstrate a positive enhancement in MRI following NAC despite the absence of residual invasive cancer. In general, HER2-positive or hormone receptor-negative tumors grow more rapidly and may require higher neovascularization than their counterparts. Although the biological aspect of the FN MRI remains unclear, tumor vasculature may remain following NAC if a primary tumor previously exhibited abundant vascularization. Tumor neovascularization at baseline and the sensitivity to NAC may be important in the mechanism of the FN MRI.

Of the 4 molecular subtypes, the present study demonstrated that sensitivity, specificity, accuracy and PPV of MRI for predicting $\mathrm{pCR}$ were the highest in triple-negative tumors. In addition, non-luminal/HER2-positive tumors revealed an extremely high pCR rate and PPV. However, these tumors were identified with the lowest NPV (38\%). As a result, MRI is concluded to be the most useful for prediction of $\mathrm{pCR}$ following NAC in patients with triple-negative tumors. When a cCR is diagnosed by MRI in patients with triple-negative tumors, the removed volume of the breast tissue must be minimized.

In this study, MRI predicted the presence or absence of residual DCIS when there were no residual invasive cancer following NAC. The accuracy of these predictions was almost the same as that of residual invasive cancer. The presence of residual DCIS following NAC is important for determining the extent of surgery necessary to achieve free surgical margins. In addition, it is important for predicting the risk of recurrence. Previously, von Minckwitz et al (8) observed that the presence of residual DCIS in the absence of invasive cancer was associated with increased recurrence risk compared with absence of residual DCIS and invasive cancer. The authors recommended that pCR must be defined as the absence of residual invasive cancer and DCIS in the breast and nodes. Accordingly, MRI is important for deciding the extent of surgery and may also be useful for estimating patient prognosis.

The present study only analyzed the status of gadolinium enhancement at each phase of MRI. Simultaneous analysis of time-signal intensity curves when judging the residual tumor may prove useful. This analysis may decrease the number of FN cases. Woodhams et al (16) reported that the accuracy for depicting residual tumors following NAC by DW-MRI is similar to that depicted by contrast material-enhanced MRI. In this study, DW images were referred for the evaluation of tumor response to NAC, however, the final diagnosis of $\mathrm{cCR}$ was based on the status of gadolinium enhancement.

In conclusion, the accuracy, PPV and NPV of MRI for predicting $\mathrm{pCR}$ following NAC were $>70 \%$. These rates differed between molecular subtypes of breast cancer and MRI was useful in predicting pCR, particularly in triple-negative tumors.

\section{Acknowledgements}

The authors thank Mr. Tomohiro Matsumoto for preparation of MRI images and technical management of MRI devices and Drs Kimito Suemasu, Jun Ninomiya, Yoshio Horii, Mari Kamimura, Miho Yoshida, Yasutaka Hagiwara, Katsunori Tozuka, Yuko Ishikawa and Toru Higuchi for performing surgical therapies.

\section{References}

1. Fisher B, Bryant J, Wolmark N, et al: Effect of preoperative chemotherapy on the outcome of women with operable breast cancer. J Clin Oncol 16: 2672-2685, 1998.

2. Rastogi P anderson SJ, Bear HD, et al: Preoperative chemotherapy: updates of National Surgical Adjuvant Breast and Bowel Project Protocols B-18 and B-27. J Clin Oncol 26: 778-785, 2008.

3. Symmans WF, Peintinger F, Hatzis C, et al: Measurement of residual breast cancer burden to predict survival after neoadjuvant chemotherapy. J Clin Oncol 25: 4414-4422, 2007.

4. Smith IC, Heys SD, Hutcheon AW, et al: Neoadjuvant chemotherapy in breast cancer: significantly enhanced response with docetaxel. J Clin Oncol 20: 1456-1466, 2002.

5. Buzdar AU, Valero V, Ibrahim NK, et al: Neoadjuvant therapy with paclitaxel followed by 5-fluorouracil, epirubicin and cyclophosphamide chemotherapy and concurrent trastuzumab in human epidermal growth factor receptor 2-positive operable breast cancer: an update of the initial randomized study population and data of additional patients treated with the same regimen. Clin Cancer Res 13: 228-233, 2007.

6. Nakamura S: Present role and future perspectives of the evaluation of the effect of primary chemotherapy by breast imaging. Breast Cancer 11: 134-138, 2004.

7. Hylton N: MR imaging for assessment of breast cancer response to neoadjuvant chemotherapy. Magn Reson Imaging Clin N Am 14: 383-389, 2006

8. von Minckwitz G, Untch M, Blohmer J-U, et al: Definition and impact of pathologic complete response on prognosis after neoadjuvant chemotherapy in various intrinsic breast cancer subtypes. J Clin Oncol 30: 1796-1804, 2012.

9. Kurosumi M, Akashi-Tanaka S, Akiyama F, et al: Histopathological criteria for assessment of therapeutic response in breast cancer (2007 version). Breast Cancer 15: 5-7, 2008.

10. Edge SB, Compton CC: The American Joint Committee on Cancer: the 7th edition of the AJCC cancer staging manual and the future of TNM. Ann Surg Oncol 17: 1471-1474, 2010.

11. Chen JH, Fieg B, Agrawal G, et al: MRI evaluation of pathologically complete response and residual tumors in breast cancer after neoadjuvant chemotherapy. Cancer 112: 17-26, 2008.

12. Chen JH, Bahri S, Mehta RS, et al: Breast cancer: evaluation of response to neoadjuvant chemotherapy with 3.0-T MR imaging. Radiology 261: 735-743, 2001.

13. Kuzucan A, Chen JH, Bahri S, et al: Diagnostic performance of magnetic resonance imaging for assessing tumor response in patients with HER2-negative breast cancer receiving neoadjuvant chemotherapy is associated with molecular biomarker profile. Clin Breast Cancer 12: 110-118, 2012.

14. McGuire KP, Toro-Burguete J, Dang H, et al: MRI staging after neoadjuvant chemotherapy for breast cancer: does tumor biology affect accuracy? Ann Surg Oncol 18: 3149-3154, 2011.

15. De Los Santos J, Bernreuter W, Keene K, et al: Accuracy of breast magnetic resonance imaging in predicting pathologic response in patients treated with neoadjuvant chemotherapy. Clin Breast Cancer 11: 312-319, 2011.

16. Woodhams R, Kakita S, Hata $\mathrm{H}$, et al: Identification of residual breast carcinoma following neoadjuvant chemotherapy: diffusion-weighted imaging-comparison with contrast-enhanced MR imaging and pathological findings. Radiology 254: 357-366, 2010. 